$10 *$ Correspondence:

.

\section{ORCID iD(s):}

3

5

7

\title{
Composition of the North American wood frog (Rana sylvatica) skin microbiome and seasonal variation in community structure
}

\author{
Alexander J. Douglas ${ }^{1}$, Laura. A. Hug ${ }^{1}$, and Barbara A. Katzenback ${ }^{1, *}$ \\ ${ }^{1}$ Department of Biology, University of Waterloo, Waterloo, Ontario, Canada, N2L 3G1
}

Dr. Barbara A. Katzenback

Department of Biology

200 University Avenue West

University of Waterloo

Phone: (519) 8884567 x 30192

E-mail: barb.katzenback@uwaterloo.ca

A.J.D. 0000-0002-6902-8701

L.A.H. 0000-0001-6171-5040

B.A.K. 0000-0001-5974-9428 


\section{Abstract}

While a number of amphibian microbiomes have been characterized, it is unclear how

31 microbial communities might vary in response to seasonal changes in the environment and the

32 behaviors which many amphibians exhibit. Given recent studies demonstrating the importance of

33 the skin microbiome in frog innate immune defenses against pathogens, investigating how

34 changes in the environment impact the microbial species present, and thus their potential

35 contribution to skin host defense, will provide a better understanding of conditions that may alter

36 host susceptibility to pathogens in their environment. We sampled the skin microbiome of North

37 American wood frogs (Rana sylvatica) from two breeding ponds in the spring, along with the

38 microbial community present in their vernal breeding pools, and frogs from the nearby forest

39 floor in the summer and fall to determine whether the microbial composition differs by sex,

40 vernal pond site, or temporally across season (spring, summer, fall). Taxon abundance data

41 reveals a profile of bacterial phyla similar to those previously described on anuran skin, with

42 Proteobacteria, Bacteroidetes, and Actinobacteria dominating the wood frog skin microbiome.

43 Our results indicate that sex had no significant effect on skin microbiota diversity, however, this

44 may be due to our limited female sample size. Vernal pool site had a small but significant effect

45 on skin microbiota, but skin-associated communities were more similar to each other than to the

46 communities observed in the frogs' respective pond water. Across seasons, diversity analyses

47 suggest there are significant differences between the skin microbiome of frogs from spring and

48 summer/fall groups while the average $\alpha$-diversity per frog remained consistent. Bacterial genera

49 known to have antifungal properties such as Pseudomonas spp. and Rhizobium spp. were

50 prevalent, and several were considered core microbiota during at least one season. These results

51 illustrate seasonal variation in wood frog skin microbiome structure and highlight the importance 
52 of considering temporal trends in an amphibian microbiome, particularly for species whose life

53 history requires recurrent shifts in habitat and behavior.

54 Keywords: Microbiome, Amphibian, Rana sylvatica, Skin, Innate Immunity, Season 


\section{Introduction}

Amphibians have unique communities of skin-dwelling microbes regulated by various mechanisms, including inoculation from the environment, skin-sloughing and specific skin secretions $[1,2]$. Studies of the amphibian skin microbiome have revealed that while many

59 bacterial phyla are present, species from Acidobacteria, Actinobacteria, Bacteroidetes,

60 Cyanobacteria, Firmicutes and Proteobacteria tend to dominate [1, 3-5]. Many families and

61 genera within these phyla are present on a wide range of amphibian species, but the specific

62 species tend to be unique to the host. Additionally, species which dominate the skin-associated

63 community are not generally those which are dominant in the environment at large, and therefore

64 must be selected for by the skin microenvironment [1]. Further microbiome studies have shown

65 that frogs of the same species sampled during different seasons or from different habitats can

66 have large differences in the diversity of bacteria present on their skin $[4,6,7]$, underscoring the

67 influence of environmental factors on the microbial community. Most studies of amphibian

68 microbiota have been performed on amphibian species from tropical or subtropical climates,

69 meaning data on temperate amphibian species is less comprehensive. Therefore, it is currently

70 unclear how stronger seasonal differences in climate and host behavior might influence the

71 amphibian skin microbiome.

Although the functional significance of amphibian skin microbiota are not yet well

73 defined, recent studies have highlighted the importance of the skin commensal microbiome to the

74 amphibian host skin defense mechanisms against invading pathogens [2, 8-10]. Through the

75 production of antifungal metabolites [11], some bacterial species commonly found on the skin of

76 amphibians inhibit the growth of the pathogenic fungi Batrachochytrium dendrobatidis [12, 13],

77 the causative agent of amphibian chytridiomycosis and proximate cause of amphibian declines 
78 on multiple continents $[14,15]$. Transfer of these specific bacteria to the skin of salamanders

79 suffering from chytridiomycosis reduced the severity of symptoms [16], which made a strong

80 case for the protective effects of select commensal bacterial species and has spurred the use of

81 bacterial species as probiotic washes for target amphibian populations [17]. However, the

82 effectiveness of specific bacteria to inhibit infection has been shown to vary based on factors

83 such as the strains of the symbiont and pathogen as well as temperature [18], so it is unlikely that

84 any single species of bacteria would provide universal protection. Additionally, pathogen

85 infection drives changes in the structure of bacterial communities on the skin and correlates with

86 lower community diversity [19-21]. It is increasingly clear that the characteristics of the

87 amphibian skin microbiome are related to infection status and that the structure and diversity of

88 the commensal microbial community may be key to predicting pathogen resistance. Thus,

89 understanding how frog skin microbial community composition varies across environmental

90 conditions would provide important information in predicting environments where amphibians

91 may be more or less susceptible to pathogens.

92 The North American wood frog (Rana sylvatica) is widespread with a range extending

93 through most of Canada, Alaska, and the Northeastern United States [22]. R. sylvatica inhabits

94 uplands environments and the far north where few, if any, other frog species inhabit. Wood frogs

95 breed in temporary pools in the early spring, leave these pools to migrate upland into the

96 terrestrial forest environment during warmer months [23] and hibernate on the forest floor in

97 winter, routinely surviving multiple sustained bouts of whole body freezing [24, 25]. R. sylvatica

98 is susceptible to infection by Frog Virus 3 and $B$. dendrobatidis [26, 27], pathogens which

99 threaten amphibian populations worldwide. Despite the wide range of $R$. sylvatica and its known

100 susceptibility to pathogens of concern, no comprehensive study of skin microbiota has ever been 
101 performed on this species. Given the seasonal shifts in habitat and behavior experienced by $R$.

102 sylvatica, they are well-suited to investigate trends which may apply to other temperate species,

103 such as whether seasonal changes in microbiome composition might lead to windows of

104 particular protection from, or susceptibility to, amphibian pathogens, as has been explored in

105 other species [20, 28, 29].

106 The objectives of this study are to (1) identify the composition of the $R$. sylvatica skin

107 microbiome and (2) determine whether the skin microbiome community structure varies with

108 sex, vernal pool of origin or across seasons. It is hypothesized that the wood frogs carry a range

109 of bacterial phyla similar to other frog skin microbiomes, with variation in representation and

110 abundance of bacterial taxa between individuals to reflect separate ponds of origin and season of

111 capture. To determine whether this is the case, we have analyzed the microbial community

112 present on the skin of $R$. sylvatica using 16 S rRNA gene amplicon sequencing. Skin swabs were

113 obtained from frogs captured from two spatially separated ponds in the spring, and the

114 surrounding forest in summer and fall so that skin-associated microbial communities might be

115 compared on a seasonal basis, as well as between different capture sites.

\section{Materials and Methods}

\section{$117 \quad 2.1 \quad$ Experimental Design \& Sample Collection}

118 Wild $R$. sylvatica adults were sampled from a site in the Waterloo Region of Ontario,

119 Canada, during the spring (April - May), summer (July - August) and fall (October). In the

120 spring, individuals were captured from two vernal ponds spatially separated by $200 \mathrm{~m}$, herein

121 referred to as Pond 1 and Pond 2, while individuals were captured from the surrounding forest

122 floor during the summer and fall seasons. Frog skin microbiota sample sizes were dependent on 
123 the number of frogs that could be captured successfully. Sampling numbers are given for each

124 site and season in Table 1.

125 Individuals were captured by nets and each frog was handled with a new pair of sterile

126 nitrile gloves. Wood frogs were gently rinsed with sterile distilled water to remove transient

127 microbes. To collect resident microbes frogs were swabbed with a sterile rayon-tipped applicator

128 (Puritan Medical Products Company, LLC., Guilford, ME, USA) 12 times on both the dorsal and

129 ventral surfaces, covering as much of the skin surface as possible. To control for environmental

130 microbes that may have deposited onto the swab during the sampling process, "field control"

131 swabs were produced by wetting a clean rayon swab with sterile distilled water and mimicking

132 the swabbing action in the open air. Each rayon swab head was placed into a sterile $1.7 \mathrm{~mL}$

133 microfuge tube and the applicator stick cut just above the rayon tip using flame-sterilized

134 scissors. Samples were transported on ice prior to storage at $-80{ }^{\circ} \mathrm{C}$. Animal care and handling

135 was performed in accordance with the guidelines of the University of Waterloo Animal Care

136 Committee and the Canadian Council on Animal Care (Animal Utilization Projects \#30008 and

137 \#40721), and animals captured under the Ontario Ministry of Natural Resources and Forestry

138 Wildlife Scientific Collectors Authorization Permits (\#1088586 and 1092603) issued to Dr. B.A.

139 Katzenback.

When individuals were captured from vernal pools, a water sample was taken by pushing

$14150 \mathrm{~mL}$ of pond water, taken from just below the surface of the water, through a Sterivex-GP PES

$1420.22 \mu \mathrm{m}$ filter (Millipore, Burlington, MA, USA) using a $50 \mathrm{~mL}$ syringe (Fisher). The filter units

143 were disconnected from the syringe, placed in individual sterile $50 \mathrm{~mL}$ conical tubes (FroggaBio)

144 and held on ice prior to storage at $-80{ }^{\circ} \mathrm{C}$. 


\subsection{DNA Isolation and Amplicon Sequencing}

DNA isolation was performed using the DNeasy PowerSoil Kit (QIAGEN Inc., Venlo, Netherlands) according to the manufacturer's protocol. Frog skin swab samples or field controls were removed from $-80{ }^{\circ} \mathrm{C}$ and immediately transferred from their storage tubes into PowerBead tubes. DNA was isolated from vernal pool microbiota filtride by removing the filter paper from the cartridge and cutting the filter paper into thin strips using flame-sterilized scissors before addition to the PowerBead tubes. To control for bacterial contamination from the laboratory environment and/or the extraction kit components, a clean rayon swab was wet with sterile distilled water and cut into a labelled $1.7 \mathrm{~mL}$ microfuge tube with flame sterilized scissors to act as a "process control" and was processed alongside the samples. All samples (skin swabs, vernal pool filters, field controls, process controls) were immediately vortexed for $10 \mathrm{~s}$ after transfer to the PowerBead tubes prior to following the manufacturer's protocol. Isolated DNA was eluted in the provided elution buffer and stored at $-80{ }^{\circ} \mathrm{C}$.

Presence of bacterial DNA was confirmed using PCR amplification. Each reaction contained $18.875 \mu \mathrm{L}$ of molecular biology grade water, $2.5 \mu \mathrm{L}$ of $10 \times$ PCR buffer, $0.5 \mu \mathrm{L}$ of 10 $\mu \mathrm{M}$ dNTPs, $1 \mu \mathrm{L}$ of $10 \mu \mathrm{M}$ forward primer 515FB 5'-GTGYCAGCMGCCGCGGTAA-3' [30, 31], $1 \mu \mathrm{L}$ of $10 \mu \mathrm{M}$ reverse primer 926R 5'-CCGYCAATTYMTTTRAGTTT-3' [30, 32], and $0.125 \mu \mathrm{L}$ Taq DNA polymerase (5 units/ $\mu \mathrm{L}$ ) (GeneDirex) per $1 \mu \mathrm{L}$ of sample, for a total reaction volume of $25 \mu \mathrm{L}$. PCR was performed with the following cycling conditions: $94{ }^{\circ} \mathrm{C}$ for $3 \mathrm{~min}$, then 35 cycles of $94{ }^{\circ} \mathrm{C}$ for $45 \mathrm{~s}, 50{ }^{\circ} \mathrm{C}$ for $1 \mathrm{~min}$ and $72{ }^{\circ} \mathrm{C}$ for $1.5 \mathrm{~min}$, followed by extension at $72{ }^{\circ} \mathrm{C}$ for $5 \mathrm{~min}$. PCR product were separated by electrophoresis at $130 \mathrm{~V}$ for $30 \mathrm{~min}$ on $2 \%$ agarose gel in TAE buffer and visualized using SafeRed dye and trans-UV (302 nm) imaging in a ChemiDoc XRS+ (Bio-Rad). 
Samples were sent for $16 \mathrm{~S}$ rRNA gene amplicon sequencing by MetagenomBio

169 (Waterloo, ON, Canada). PCR reactions were prepared in triplicate for each sample. Each $25 \mu \mathrm{L}$

170 reaction mixture contained $1.6 \mu \mathrm{L}$ of molecular grade water, $0.2 \mu \mathrm{L}$ of BSA $(20 \mathrm{mg} / \mathrm{mL}), 2.5 \mu \mathrm{L}$

171 of $10 \times$ standard Taq buffer, $0.5 \mu \mathrm{L}$ of $10 \mathrm{mM}$ dNTPs, $5.0 \mu \mathrm{L}$ of $1 \mu \mathrm{M}$ forward primer $515 \mathrm{FB} 5^{\prime}-$

172 GTGYCAGCMGCCGCGGTAA-3' [30, 31], $5.0 \mu \mathrm{L}$ of $1 \mu \mathrm{M}$ reverse primer 806RB 5'-

173 GGACTACNVGGGTWTCTAAT-3' [30, 31], $0.2 \mu \mathrm{L}$ of Taq DNA polymerase (5 units/ $\mu \mathrm{L}$ ), and

$17410 \mu \mathrm{L}$ of sample DNA. PCR was performed with the following thermocycling conditions: $95^{\circ} \mathrm{C}$

175 for $5 \mathrm{~min}, 35$ cycles of $95^{\circ} \mathrm{C}$ for $30 \mathrm{~s}, 50^{\circ} \mathrm{C}$ for $30 \mathrm{~s}$ and $72^{\circ} \mathrm{C}$ for $60 \mathrm{~s}$, followed by an extension

176 at $72^{\circ} \mathrm{C}$ for $10 \mathrm{~min}$. The products of the triplicate reactions were pooled and resolved with $2 \%$

177 TAE agarose gel. PCR products of the correct amplicon size ( $291 \mathrm{bp})$ were excised, pooled,

178 gel purified and quantified using the Invitrogen ${ }^{\mathrm{TM}}$ Qubit $^{\mathrm{TM}} \mathrm{dsDNA}$ HS Assay Kit (Thermo Fisher

179 Scientific Inc., Waltham, MA, USA). For all 2018 samples sequencing was performed using an

180 Illumina MiSeq and the MiSeq Reagent Kit v2 (Illumina, Inc., San Diego, CA, USA) for 2 sets

181 of 250 cycles. This was increased to 3 sets of 250 cycles for all 2019 samples due to an error at

182 the sequencing center

$183 \quad 2.3$ Amplicon Sequence Data Processing

184 Sequence data was obtained as FASTQ files in the CASAVA 1.8 paired-end

185 demultiplexed format. Files from repeat sequencing runs were concatenated to create a FASTQ

186 file containing all of the observed sequences for each sample. These files were imported into

187 QIIME 2 v2019.1.0 [33] and all analysis was performed using QIIME 2 unless otherwise stated.

Using DADA2 [34], reads were trimmed by 25 bp on the 5' end to remove the primer

189 sequence and truncated to 245 bp to remove low quality regions, filtering out any reads shorter

190 than this length. The reads were dereplicated, denoised and any chimeric sequences were 
191 removed. Paired forward and reverse reads were merged, generating the final amplicon sequence

192 variants (ASVs). Each ASV was assigned taxonomy using a naïve Bayesian classifier trained on

193 the SSU Ref NR 99 dataset from the SILVA 132 release [35], with sequences trimmed to include

194 only the V4-V5 region. All unassigned ASVs and those assigned to chloroplast or mitochondria

195 were filtered from the samples. To minimize erroneous ASVs, a minimum frequency of 22

196 (0.001\% of the total sequence count) was set, and any ASVs with a total frequency less than 22

197 were filtered from the samples.

\subsection{Taxonomic Assignment and Significance}

A multiple sequence alignment (MSA) was produced from the ASVs using MAFFT [36].

200 Columns of the alignment which were ambiguously aligned were masked to avoid introducing

201 error to the phylogenetic model. A phylogenetic tree was generated from the MSA using

202 RAxML [37] with 100 bootstraps. The RAxML tree was assigned a midpoint root and used for

203 all further phylogenetic analysis.

Core taxa were determined for each group of seasonal frog skin communities, and for the overall frog skin microbiome, using the core-features command in QIIME2. Core taxa were

206 defined as those found to be present in $90 \%$ or more of the samples within a given group, as this

207 is a commonly used threshold [6, 38] which meets the definition of core microbiota as those

208 which are commonly present within samples from a given environment [39]. Venn diagrams

209 depicting the overlap of seasonally core microbiota were prepared using the online Venn

210 diagram tool provided by the University of Ghent

211 (http://bioinformatics.psb.ugent.be/webtools/Venn/). 
To assess the presence of microbiota with putative antifungal activity, bacterial taxa

213 identified from the R. sylvatica skin microbiome were searched against the Antifungal Isolates

214 Database [40]. The metadata file for the Antifungal Isolates Database was obtained and sorted

215 based on genera, filtering for those genera which were detected on $R$. sylvatica skin. Genera with

216 one or more antifungal isolates were considered putatively antifungal, and their relative

217 abundances in the wood frog skin microbial community were compared between seasons.

\section{$218 \quad 2.5 \quad$ Statistical Analyses}

219 For diversity analyses, samples were rarified to 10,000 sequences. Those containing less

220 than 10,000 were omitted from diversity analyses. The phylogenetic tree and rarified ASV

221 frequencies were used to calculate various $\alpha$-diversity metrics (Faith's Phylogenetic Diversity,

222 Shannon's Diversity Index, ASV richness). Diversity was compared between groups using a one-

223 way analysis of variance (ANOVA).

224 To assess differences in microbial community composition between samples, various $\beta$ -

225 diversity metrics were calculated (Unweighted UniFrac, Weighted UniFrac and Bray-Curtis

226 dissimilarity). $\beta$-diversity was visualized using principal coordinate analysis (PCoA). The adonis

227 function from the $\mathrm{R}$ vegan package was used to perform permutational multivariate analysis of

228 variance (PERMANOVA) with 999 permutations. To determine whether groups had significant

229 differences in microbiome composition, pairwise PERMANOVA tests were applied to the

230 dissimilarity matrix produced by each $\beta$-diversity metric and performed with 999 permutations. 


\section{Results}

\section{$233 \quad 3.1 \quad$ Microbiome Overview Statistics}

234 We obtained microbial 16S rRNA amplicon sequences from each of the 66 frog swabs, 5

235 water samples, 5 field blanks, and 3 process blanks. After filtering based on quality, taxonomy

236 and minimum frequency, a total of 1,937,866 sequences remained. Sequence counts varied

237 between samples considerably, and those samples sequenced in the later set (Spring 2019 frog

238 swabs, water samples and associated blanks) had much higher counts on average due to their

239 increased sequencing depth. A total of 4,325 ASVs were recognized, which ranged in frequency

240 from 22 to 159,313 appearances, with a median frequency of 60 . These ASVs were matched to

$241 \quad 1,384$ unique taxonomic assignments, 1,123 of which were specific to at least the genus level.

We found that DNA isolated from process controls yielded ASVs belonging to 9 bacterial

243 phyla and contained 37 ASVs each on average, which equated to a total of 71 unique taxa when

244 combined at the genus level (Supplementary Table 1). Unlike the communities found on frog

245 skin and in pond water, the majority of ASVs found in the process controls belonged to a limited

246 group of genera, primarily Curtobacterium (43\%), with Lactobacillus (16\%) and Acholeplasma

247 (7.7\%) also more abundant. The field controls generally had more diverse communities, with an

248 average of 64 ASVs per blank. These ASVs belonged to a total of 15 phyla, and 164 unique taxa

249 when combined at the genus level (Supplementary Table 2). The genera with the greatest mean

250 relative abundance was once again Curtobacterium (8.4\%), but genera which were abundant on

251 frog skin such as Massilia and Pseudomonas were also found to be abundant in the field blanks

252 (4.1\% and 3.8\%, respectively). On average 11\% of amplicons detected in frog skin samples and

$2530.07 \%$ of amplicons detected in water samples were ASVs which were also found in the process

254 controls, while an average of $35 \%$ of amplicons detected in frog skin samples and $32 \%$ of 
amplicons detected in water samples were ASVs which were also found in the field controls. Of

256 the $11 \%$ of the amplicons found on frogs which were shared with the process blank, the majority

257 (5.8\% of mean relative abundance) corresponded to a single ASV belonging to Curtobacterium.

258 A large portion of the ASVs belonging to Curtobacterium were found on a group of five frogs

259 where this ASV made up more than $50 \%$ of amplicons detected.

260

261

262

263

264

265

266

267

268

269

270

271

272

273

274

275

276

\subsection{Capture Site Influences the Structure of Microbial Communities}

Spring was the only season in which sampling occurred across two distinct sites, the two vernal pools. It was thus necessary to investigate how pond of origin might influence an individual's skin microbiome. We observed that the skin microbiota of wood frogs captured from Pond 1 and Pond 2 had very similar $\alpha$-diversity metrics, and both of the skin-associated communities had higher average microbial diversity than the microbial communities present in the water samples taken from either vernal pond (Fig. 1). When we sorted frog skin microbiota and vernal pond microbiota samples from spring 2018 and spring 2019 into groups based on their pond of origin, there were significant differences in $\alpha$-diversity (ANOVA: ASV richness: $p$ $=0.0017$; Shannon diversity index: $\mathrm{p}=0.0032$; Faith's phylogenetic diversity: $\mathrm{p}=0.0041$ ). Differences were between the Pond 2 water and the Pond 1 frog skin (pairwise ANOVA: ASV richness: $\mathrm{p}=0.0355$; Shannon diversity index: $\mathrm{p}=0.0629$; Faith's phylogenetic diversity: $\mathrm{p}=$ 0.0068) and between Pond 2 water and Pond 2 frog skin (pairwise ANOVA: ASV richness: $\mathrm{p}=$ 0.0029; Shannon diversity index: $\mathrm{p}=0.0114$; Faith's phylogenetic diversity: $\mathrm{p}=0.0035$ ). There were no significant differences in community diversity between Pond 1 water and Pond 1 frog skin, Pond 1 water and Pond 2 frog skin, Pond 1 water and Pond 2 water or Pond 1 frog skin and Pond 2 frog skin. 
The microbial communities present in each group of frog skin samples and water samples

278 included many of the same bacterial phyla and had a high proportion of ASVs belonging to

279 Proteobacteria, Bacteroidetes and Actinobacteria (Fig. 2). These three phyla were the only phyla

280 we found to be highly abundant in the water samples, while all other phyla present had a mean

281 relative abundance of less than $1 \%$. The frog skin communities from both ponds also had

282 Acidobacteria and Verrucomicrobia ASVs present at greater than $1 \%$ mean relative abundance,

283 and Pond 2 frog skin community additionally had Firmicutes ASVs present at more than $1 \%$

284 mean relative abundance. We employed pairwise analysis of variance (ANOVA) to compare the

285 relative abundance of each of these 6 common phyla between the water samples and frog skin

286 samples from both ponds and no significant difference in abundance was observed for any of the

287 phyla. Beyond the most abundant phyla, the frog skin microbiota exhibited a more diverse range

288 of phyla compared to vernal pool microbiota; the skin-associated communities from frogs

289 captured from Pond 1 and Pond 2 included ASVs from phyla that were not present in any pond

290 water samples.

Sample type (frog skin microbiota, vernal pond microbiota), site (Pond 1, Pond 2) and

292 year all had significant $(\mathrm{p}<0.005)$ but small $\left(\mathrm{R}^{2}<0.12\right)$ effects on community composition and

293 abundance of the observed taxa when assessed using adonis (Table 2). Sample type had the

294 largest effect, particularly when considering abundance as observed with Weighted UniFrac

295 distance (Table 2b, adonis pseudo- $\mathrm{F}=6.86, \mathrm{p}<0.001, \mathrm{R}^{2}=0.11$ ) and Bray-Curtis dissimilarity

296 (Table 2c, adonis pseudo-F $=7.71, \mathrm{p}<0.001, \mathrm{R}^{2}=0.12$ ). Effect of the sample type (Table 2a,

297 adonis pseudo-F $\left.=4.08, \mathrm{p}<0.001, \mathrm{R}^{2}=0.07\right)$ and year $($ Table $2 \mathrm{a}$, adonis pseudo-F $=4.20, \mathrm{p}<$

$2980.001, \mathrm{R}^{2}=0.07$ ) had equal effects as observed with Unweighted UniFrac distance. Principal

299 coordinate analysis of the $\beta$-diversity metrics did not reveal clear clustering of frog skin 
microbiota by pond origin under any conditions (Fig. 3). Water samples were found to cluster separately from frog samples only when relying on Bray-Curtis dissimilarity values (Fig. 3c) and were not clearly distinguished from frog samples when considering phylogenetic diversity (Fig. from Ponds 1 and 2 were largely similar, we combined these groups in further analysis.

\subsection{Host Sex Does Not Affect Diversity and Structure of Microbial Communities}

Due to sampling limitations, very few female frogs $(n=8)$ were included compared to the

$308=6$ ) were captured during spring, meaning that any sex-dependent microbiome characteristics

311 frogs using a t-test with Welch's correction resulted in no significant difference for any metric

312 observed (ASV richness: $p=0.3863$; Shannon diversity index: $p=0.2717$; Faith's phylogenetic

313 diversity: $\mathrm{p}=0.4585)$. Additionally, sex was not a significant driver of community structure

314 when assessed using adonis for the three $\beta$-diversity metrics used (Unweighted UniFrac: pseudo-

$315 \mathrm{~F}=1.13, \mathrm{p}=0.241, \mathrm{R}^{2}=0.019 ;$ Weighted UniFrac: pseudo- $\mathrm{F}=1.83, \mathrm{p}=0.097, \mathrm{R}^{2}=0.031$;

Bray-Curtis: pseudo- $\mathrm{F}=1.30, \mathrm{p}=0.147, \mathrm{R}^{2}=0.022$ ).

\subsection{Season of Capture Influences the Structure of Microbial Communities}

To assess the influence of season on the skin microbiome the frog samples were divided into groups based on capture date (Spring 2018, Summer 2018, Fall 2018 and Spring 2019). The

321 4). Of the three $\alpha$-diversity metrics we considered, two differed significantly between seasonal

322 sample groups (ANOVA: ASV richness, p < 0.0001; Faith's phylogenetic diversity, p < 0.0025). 
323 In both cases the mean diversity of the Spring 2019 group was significantly higher than that of

324 Spring 2018 and Summer 2018, while no other groups had significantly different means.

We found that community structure differed between seasonal groups, including

326 observable trends at the highest taxonomic levels (Fig. 2). While the majority of ASVs present in

327 any given sample were typically Proteobacteria, the abundance of ASVs belonging to other

328 major phyla such as Bacteroidetes, Actinobacteria, Verrucomicrobia and Acidobacteria varied

329 considerably (Fig. 2). Five samples from Spring 2019 were notable outliers, which had a unique

330 community structure not observed in other samples. These frogs were all captured on the first

331 day of sampling in Spring 2019 at Pond 1 and were the only samples where ASVs belonging to

332 Actinobacteria made up more than $40 \%$ of the sequences detected, while those belonging to

333 Proteobacteria were much less abundant (below 40\%). We assessed the variation in relative

334 abundance of the 5 major phyla between seasonal groups using one-way ANOVA. All groups

335 exhibited some seasonal variation (Acidobacteria, $\mathrm{p}<0.0001$; Actinobacteria, $\mathrm{p}=0.0138$;

336 Bacteroidetes, $\mathrm{p}=0.0345$; Proteobacteria, $\mathrm{p}=0.0002 ;$ Verrucomicrobia, $\mathrm{p}=0.0389)$.

337 Acidobacteria was the only phylum showing a clear trend, with a significantly higher mean

338 relative abundance (pairwise ANOVA: $\mathrm{p}<0.0219)$ in Summer and Fall $2018(4.7 \%$ and 6.4\%

339 respectively) than Spring 2018 and 2019 (1.9\% and 0.62\%, respectively).

Season was a significant source of variation in community structure when measured

341 using Unweighted UniFrac (Table 3a, adonis pseudo-F $=4.36, \mathrm{p}<0.001, \mathrm{R}^{2}=0.13$ ), Weighted

342 UniFrac (Table 3b, adonis pseudo-F $\left.=7.04, \mathrm{p}<0.001, \mathrm{R}^{2}=0.20\right)$ and Bray-Curtis (Table 3c,

343 adonis pseudo-F $\left.=6.17, \mathrm{p}<0.001, \mathrm{R}^{2}=0.18\right)$. We performed pairwise PERMANOVAs to

344 determine which seasonal groups had significantly different community structure and every

345 pairwise comparison revealed significant difference across all $\beta$-diversity metrics 
346 (PERMANOVA: $\mathrm{p}=0.001$ ), with the exception of the Summer and Fall 2018 frog skin

347 microbiome groups which were not significantly different when considering Weighted UniFrac

348 distance (PERMANOVA: pseudo-F = 2.60, $\mathrm{p}=0.102$ ). Summer and Fall 2018 frog skin

349 microbiomes from were found to be significantly different when considering Unweighted

350 UniFrac distance (PERMANOVA: pseudo-F $=1.69, \mathrm{p}=0.045$ ) and Bray-Curtis dissimilarity

351 (PERMANOVA: pseudo-F $=1.84, \mathrm{p}=0.045$ ), though we noted that $\mathrm{p}$ values were near-

352 threshold in both cases. This result was reflected in the Principle Coordinate Analysis results

353 (Fig. 5), where skin microbiota from frogs collected during the summer and fall tended to cluster

354 together, and appeared to form a cluster distinct from the spring samples when Unweighted

355 UniFrac (Fig. 5a) and Bray-Curtis distances (Fig. 5c) were considered, although summer and fall

356 samples were not clearly distinct from spring samples when visualizing Weighted Unifrac

357 distances (Fig. 5b).

\subsection{The Seasonal Core Microbiome}

We identified core microbiota for all frog skin samples as a whole, as well as for each

360 seasonal group separately. No ASV was present in $\geq 90 \%$ of all frog skin microbiome samples, so

361 ASVs were collapsed at the genus and family level to consider a more inclusive core

362 microbiome. A group of 7 genera were present in $\geq 90 \%$ of all frog samples, accounting for

363 approximately $11 \%$ of ASVs on the average individual (Table 4). A group of 11 families were

364 present in $\geq 90 \%$ of all frog samples, accounting for $56 \%$ of ASVs on the average individual

365 (Table 5). We analyzed the seasonal variation in abundance of the nine core families with a

366 mean relative abundance greater than $1 \%$ (Fig. 6), revealing six bacterial families with

367 significantly different mean relative abundance across seasonal groups: Beijerinckaceae,

368 Burkholderiaceae, Caulobacteraceae, Sphingobacteriaceae, Spirosomaceae and 
Xanthobacteracea (ANOVA: $\mathrm{p}<0.05$ ). In all cases but Sphingobacteriaceae, Summer and Fall 2018 abundances did not differ significantly. Similarly, Spring 2018 and 2019 significantly

371 differed only in the abundance of Burkholderiaceae.

When considering each seasonal group of skin associated communities separately, it was revealed that 25 of the 52 core genera and 16 of the 43 core families were considered core during

374 only one season (Fig. 7). Only two genera were core to all seasonal groups (Fig. 7a), indicating

375 that the number of core microbiota observed when considering all frogs together (7) was not

376 reflective of the core genera present during each individual season. The Spring 2018 and Spring

3772019 frog skin microbial communities had the most core genera in common (20), while Summer

3782018 and Fall 2018 had the second highest level of overlap (7). Additionally, the frog skin

379 microbiota from Spring 2018 and Spring 2019 each had a larger number of in-season core

380 genera, with 20 in Spring 2018 and 37 in Spring 2019 as compared to 13 in Summer 2018 and 14

381 in Fall 2018. In comparison, a group of 8 families were core across all seasonal groups (Fig. 7b),

382 but the consistent prevalence of these families was not always matched with a high relative

383 abundance. We found that Burkholderiaceae was the only family to consistently have a mean

384 relative abundance above $10 \%$ on wood frog skin, ranging from 13\% in Fall 2018 to $44 \%$ in

385 Spring 2018. Interestingly, there were 9 core families unique to the Fall 2018 wood frog skin

386 group, while other seasonal groups had only 2-3 uniquely core families. Fall 2018 wood frog

387 microbiomes also had the most core families overall at 26, followed by microbiota on wood frog

388 skin in Spring 2019 with 25, Summer 2018 with 21, and Spring 2018 with 19. In all seasons

389 except for Spring 2018, the core families present on wood frog skin were members of the phyla

390 Actinobacteria, Bacteroidetes, Proteobacteria and Verrucomicrobia. The core families present on

391 the skin of wood frogs in Spring 2018 lacked any members of Actinobacteria but included a 
392 single representative from the Gemmatimonadetes. In all cases the majority of core families

393 present on wood frog skin were from the Proteobacteria, which ranged from $44 \%$ to $68 \%$

394 summed relative abundance. In general, community structure appeared to be more consistent for

395 microbial families than for individual genera.

3.6 Putative Antifungal Taxa Are Present on the Skin of Wood Frogs Across Seasons

As recent studies have identified the importance of key bacterial species in protection of

398 amphibians against $B$. dendrobatidis through the production of antifungal metabolites, we

399 surveyed the wood frog microbiome for the presence of these putative antifungal bacterial

400 species. Of the 37 bacterial genera found in the Antifungal Isolates Database that have isolated

401 representatives demonstrating antifungal properties, 33 were present on $R$. sylvatica skin. The

402 core genera from each seasonal group included putatively antifungal genera with several among

403 the most prevalent genera overall, including Pseudomonas, Massilia, and

404 Allo/Neo/Para/Rhizobium. Antifungal-associated genera varied in their relative abundances

405 between wood frog skin microbiota samples, and mean relative abundances typically varied

406 widely across seasons (Table 6).

\section{Discussion}

408 As we continue to improve our understanding of the skin microbiome and its role in

409 maintaining the health of amphibians it is important to consider the inherent variability in

410 microbial communities and the factors that drive this variability. While amphibians found in

411 warmer regions may experience a relatively stable environment year-round, the majority of

412 North America amphibians experience major seasonal fluctuations in environmental conditions.

413 Changes in environmental conditions are known to influence the microbiome [4, 6, 7], 414 potentially affecting the hosts ability to resist infection by a pathogen $[18,19]$. In this study we 
415 have reported on the microbial community associated with $R$. sylvatica skin over the course of

416 multiple seasons to observe the changes in community composition and structure. To provide

417 perspective for these seasonal effects, we have compared them to the effects of host sex and

418 vernal pool site of capture within a single season (spring). Lastly, we have highlighted members

419 of the wood frog skin microbial community that are reported in the Antifungal Isolates Database

420 to produce molecules with antifungal activity, to tentatively predict the impact of season on the

421 potential ability of the skin microbiome to contribute to defense against fungal pathogens.

\section{Microbial Community Structure and Core Taxa}

We observed that the microbial community associated with $R$. sylvatica skin has much in

424 common with those found on other frog species. ASVs belonging to Proteobacteria,

425 Actinobacteria, Bacteroidetes, Firmicutes, Verrucomicrobia and Acidobacteria made up the vast

426 majority of sequences observed from all samples, suggesting that these phyla dominate the

427 microbiome. These phyla are commonly present on the skin of other frog species [3-5, 8] and all

428 but Verrucomicrobia are abundant elements of the microbial communities associated with the

429 related frog species Rana pipiens and Rana catesbeiana [1, 41]. While these phyla varied in

430 abundance on the skin of individual wood frogs, there were very few cases in which any of the

431 above bacterial phyla were found to be absent from the wood frog skin microbiota. Communities

432 were much less consistent at finer taxonomic levels and among the hundreds of microbial genera

433 observed, very few were prevalent enough to be considered core taxa. The most abundant of

434 these core genera, Sphingomonas and Pseudomonas, are widespread in the environment at large

435 and have been shown to be similarly abundant on the skin of other frogs $[1,6,41]$.

436 Many of the core taxa associated with the skin microbiome are also known contaminants

437 of commercial DNA extraction kits [42]. To better understand the extent to which microbial 
contaminants introduced during the extraction process contributed to the microbial communities

439 observed in our samples, we looked for ASVs present in both the samples and the process

440 controls. While the majority of our frog samples $(n=43)$ had a low abundance $(>5 \%$ relative

441 abundance) of ASVs which were found in the process controls, a group of seven frog skin

442 samples from Summer 2018 and Spring 2019 had very high (>50\%) relative abundance of ASVs

443 found in the process controls, which seems to indicate a high level of process contamination in

444 these samples. Unexpectedly, all water samples had a very low abundance of ASVs found in the

445 process control ( $0.07 \%$ average relative abundance) and completely lacked the Curtobacterium

446 ASV which was highly abundant in process blanks, field blanks and frog samples. This suggests

447 that potential contamination from reagents was not universal, or that many of the ASVs detected

448 in the process controls were also naturally present on the frogs and surrounding environment.

449 Additionally, samples with low numbers of detected amplicons did not have a proportionally

450 higher relative abundance of ASVs found in the process controls, as would be expected of a

451 failed skin swab which did not capture frog skin microbiota. Overall, while contamination from

452 reagents and exposure to the laboratory environment was impossible to avoid, it does not appear

453 to contribute to the observed trends in community structure.

\section{$454 \quad 4.2$ Effect of Vernal Pool Site and Host Sex on the Wood Frog Skin Microbiome}

455 While $R$. sylvatica are generally terrestrial and solitary, wood frogs converge on vernal

456 pools during the spring thaw to seek mates and reproduce [23]. In this study the two temporary

457 ponds from which frogs were captured during the spring served as the only truly distinct

458 sampling sites, since the surrounding area was fairly uniform mixed woodland. As $R$. sylvatica

459 are known to venture as far as $1 \mathrm{~km}$ from their breeding pond and the ponds sampled are $\sim 200 \mathrm{~m}$

460 apart it is unlikely that they harbor genetically distinct populations [43], and therefore any 
461 variation in the skin-associated microbial community is better attributed to the environmental

462 conditions of the site. This is an important distinction, as it is not well understood to what degree

463 host phylogenetics and environment affect the microbiome of amphibians, and examples exist

464 which emphasize the role of both factors [44-46]. Pond of origin was found to explain a small,

465 but significant amount of the variation observed in the microbial communities on frogs captured

466 during the spring. This effect was less pronounced than the variation between frogs captured in

467 spring of 2018 and 2019 however, and the largest differences were observed between frog swabs

468 and water samples. This was expected, as previous studies have established that amphibians have

469 communities of skin-associated microbiota distinct from their environment [1, 41, 47]. There

470 was no clear trend linking frog skin microbiomes to the microbiota found in the associated pond

471 water. There was no significant difference in ASV richness, evenness or phylogenetic diversity

472 between the skin-associated microbial communities in Pond 1 and Pond 2. Despite water samples

473 from Pond 2 having a lower mean ASV richness than those of Pond 1, frogs from Pond 2 had

474 more ASVs on average, suggesting that seeding of microbes from the water was not a major

475 driver of skin microbiome diversity. Frogs from both ponds hosted bacterial phyla that were

476 uncommon, or not present, in the pond water and exhibited more diverse and even communities,

477 while water samples were almost entirely populated by Proteobacteria, Bacteroidetes and

478 Actinobacteria. Given the increased abundance of many of these phyla on frogs captured during

479 the summer and fall, is seems they must either be stable members of the microbiome or are

480 seeded from rich microbial communities found in the soil and leaf litter of the surrounding forest.

481 It is unclear whether seeding from soil environments might occur while buried during winter

482 hibernation, and a study of the microbial communities present in frog hibernacula, although

483 challenging, would be an interesting avenue of future research. 
485 sex and skin microbiota have been observed in humans [48] and other vertebrates [49], but the

486 effect of sex has not been well studied in amphibians. We found sex had no significant effect on

487 structure or diversity of the microbial community. The few previous studies considering the

488 effect of sex in amphibians failed to find significant differences between males and females [21,

$48944]$, and our work, although limited by the low number of female frogs, corroborates these 490 findings.

\subsection{Effect of Season on the Wood Frog Skin Microbiome}

Season was also associated with significant variation in the structure and composition of

493 the wood frog skin microbiomes studied. The effect of season was more pronounced than the

494 effects of site, year or sample type observed in the spring samples, and was evidenced by shifts

495 in the abundance of major phyla on wood frog skin. Most notably, relative abundance of 496 Acidobacteria was significantly higher among frogs captured in the summer and fall than those 497 captured during spring. Given the particularly high abundance of Acidobacteria in soils [50] it is 498 not surprising that members of this phylum would be highly abundant on frogs active in soil and

499 leaf litter. The seasonality to Acidobacteria on frog skin matches a proposal that Acidobacteria 500 are transiently associated with the human skin microbiome [51]. While the composition of the 501 frog skin microbiome varied, average diversity of individual $R$. sylvatica microbiomes remained 502 fairly constant across seasons. When considering ASV richness and phylogenetic diversity the 503 Spring 2019 wood frog skin microbiota group was determined to have a mean diversity 504 significantly different from Spring 2018 and Summer 2018. However, when considering the 505 Shannon Diversity Index, wood frog skin microbiomes sampled during Spring 2019 falls well 506 within the range of these groups. This suggests that the Spring 2019 wood frog microbial 
communities were not as even, and a larger proportion of uncommon ASVs were contributing to

508 their diversity. This is likely at least partially the result of the Spring 2019 samples undergoing

509 an additional sequencing replicate. The resultant greater sequencing depth would increase the

510 number of rare ASVs [52], and contribute to increased observed diversity. Rarefaction prior to

511 diversity analyses was conducted to mitigate this issue, but it is not a perfect method [53].

Due to the much higher number of wood frog microbiome samples collected during the

513 spring months (75\% of total frog swab samples), any analysis considering overall prevalence of

514 taxa was heavily biased toward taxa which were common during the spring. To better represent

515 the microbial communities present on wood frog skin during summer and fall samples, core taxa

516 were considered for each seasonal group individually and overlaps in seasonal groups' core taxa

517 determined. As core taxa represent the microbes which are most commonly found in the frog

518 skin environment and often represent key members of the microbial community [39], common

519 core taxa should reflect similar community dynamics. The skin of $R$. sylvatica hosted only a

520 small number of core microbiota, particularly at lower taxonomic levels. Several of the core taxa

521 are known to be core to other frog skin communities, Pseudomonas being one of the most

522 commonly represented [6,38,41], but the majority of core taxa appeared to be fairly unique.

523 Additionally, many of the most prevalent taxa experienced significant changes in abundance

524 between seasons. The families Beijerinckiaceae and Xanthobacteraceae were significantly more

525 abundant in summer and fall, while Sphingobacteriaceae greatly increased in abundance during

526 the summer only. The variability in the core taxa observed on $R$. sylvatica skin suggests that the

527 skin microbiome is a highly dynamic environment, where seasonal factors can re-shape the core

528 structure and few 'microbes are suited to inhabit the skin year-round. 


\subsection{Seasonal Representation of Putatively Anti-fungal Microbes}

532 focused on members of the frog skin microbial community that protect against $B$. dendrobatidis.

533 Notably, we observed members of the genus Janthinobacterium on R. sylvatica skin, however it

534 was not determined whether the ASV detected belonged to the protective species

535 Janthinobacterium lividum [10, 17]. Janthinobacterium were most abundant in Spring 2018 and

536 2019, were present on only one Summer 2018 frog and were entirely absent in Fall 2018.

537 Additionally, members of the family Sphingobacteriaceae were found on frogs in all seasons.

538 This is notable as presence of Sphingobacteriaceae was a predictor of successful recovery from

539 B. dendrobatidis infection in other frog species [28]. Several other core taxa present on wood

540 frog skin have known isolates which inhibit $B$. dendrobatidis listed in the Antifungal Isolates

541 Database of amphibian skin-associated bacteria [40]. Burkholderiaceae and Xanthomonadaceae,

542 in particular, have many genera with anti- B. dendrobatidis isolated members. Among the core

543 genera, Pseudomonas and Rhizobium have the strongest evidence for $B$. dendrobatidis inhibition

544 in vitro [40]. Aside from a spike in Burkholderiaceae abundance in Spring 2018, putatively anti-

545 B. dendrobatidis taxa were not associated with any season in particular, and most were present at

546 low abundance throughout the year. While these findings do not confirm that the bacterial taxa

547 observed on $R$. sylvatica have antifungal activity, the organisms in our dataset associated with

548 these groups are of interest as putatively anti-B. dendrobatidis. Further investigation is required

549 to determine whether the specific microbial strains present on $R$. sylvatica possess anti-pathogen

550 qualities to better understand the functional significance of seasonal variation in the skin

551 microbiome and its contribution to defense against pathogens. 


\section{2}

553

554

555

556

557

558

559

560

561

562

563

564

565

566

567

568

569

570

571

572

573

574

\section{Conclusions}

Our results indicate that season has a significant effect on the structure of the North American wood frog skin microbiome and has a proportionally greater effect than spring breeding pond association. Frogs captured during summer and fall were the most similar in terms of $\beta$-diversity distances and could be distinguished from spring frogs by their increased abundance of Acidobacteria, as well as other soil-associated bacterial families. It remains unclear whether the shift towards increased abundance of soil-associated bacteria on frog skin in the summer and fall is a result of transient colonization from frequent exposure, or a stable equilibrium shift in the community. Skin-associated microbial communities had consistent structural similarities at the highest taxonomic levels but displayed a high degree of diversity at finer levels, and the few core genera identified were not a dominant component of the community. Frogs captured during all seasons were host to microbes with putative anti- $B$. dendrobatidis activity, and seasonal shifts did not seem to affect the overall pool of potentially protective taxa. $R$. sylvatica is a widespread species and further study of populations from varied environments (Boreal shield, montane forest, etc.) could reveal related trends. While the effect of season has been briefly explored in other temperate frog species [4], this study provides insight into the seasonality of skin microbiome structure on amphibian species inhabiting northern environments and establishes foundational knowledge for further study of species which experience dramatic shifts in habitat and behavior between seasons.

Acknowledgements: The authors thank Nicole Wang for the generous contribution of a trained taxonomic classifier for 16S rRNA gene sequences and Maxwell P. Bui-Marinos, Joseph F.A. Varga and Nathanael B. J. Harper for their technical assistance in collecting frog skin swabs. 
576 Author Contributions: AJD, LAH and BAK conceived the study; AJD and BAK performed

577 field sampling; AJD performed the experiments and analyzed the data; AJD, LAH and BAK

578 wrote and critically revised the manuscript.

580 Funding Information: This study was funded by a Natural Sciences and Engineering Research

581 Council of Canada Discovery Grant (NSERC DG) to BAK (Grant \# RGPIN-2017-04218) a Tier

582 II Canada Research Chair to LAH and salary support to AJD through a Natural Sciences and

583 Engineering Research Council of Canada Undergraduate Summer Research Assistantship

584 (NSERC USRA), the University of Waterloo Undergraduate Research Internship (URI) funding

585 initiative, as well as a Graduate Research Studentship, Science Graduate Award, and UW

586 Graduate Scholarship awarded by the University of Waterloo, Department of Biology.

Data Availability: 16S rRNA gene amplicon sequence data for skin microbiome samples are

589 deposited in the NCBI Sequence Read Archive (Bioproject PRJNA603391).

\section{Compliance with Ethical Standards}

593 Conflict of Interest: The authors declare that they have no conflict of interest.

595 Ethics Statement: All applicable international, national, and/or institutional guidelines for the 596 care and use of animals were followed. All procedures performed in studies involving animals 
were in accordance with the ethical standards of the institution at which the studies were

598 conducted (University of Waterloo Animal Care Committee and the Canadian Council on

599 Animal Care, Animal Utilization Projects \#30008 and \#40721; and animals captured under the

600 Ontario Ministry of Natural Resources and Forestry Wildlife Scientific Collectors Authorization

601 Permits \#1088586 and \#1092603 issued to Dr. B.A. Katzenback). This article does not contain

602 any studies with human participants performed by any of the authors.

\section{References}

605 1. McKenzie VJ, Bowers RM, Fierer N, et al (2012) Co-habiting amphibian species harbor 606 unique skin bacterial communities in wild populations. ISME J 6:588-596. https://doi.org/10.1038/ismej.2011.129

2. Colombo BM, Scalvenzi T, Benlamara S, Pollet N (2015) Microbiota and mucosal immunity in amphibians. https://doi.org/10.3389/fimmu.2015.00111

3. Walke JB, Becker MH, Hughey MC, et al (2015) Most of the dominant members of amphibian skin bacterial communities can be readily cultured. Appl. Environ. Microbiol. 81:6589-6600. https://doi.org/10.1128/AEM.01486-15

4. Longo A V., Savage AE, Hewson I, Zamudio KR (2015) Seasonal and ontogenetic variation of skin microbial communities and relationships to natural disease dynamics in declining amphibians. R. Soc. Open. Sci. 2:140377. https://doi.org/10.1098/rsos.140377

5. Belden LK, Hughey MC, Rebollar EA, et al (2015) Panamanian frog species host unique skin bacterial communities. Front. Microbiol. 6:1171. https://doi.org/10.3389/fmicb.2015.01171

6. Ellison S, Rovito S, Parra-Olea G, et al (2018) The Influence of Habitat and Phylogeny on the Skin Microbiome of Amphibians in Guatemala and Mexico. Microb. Ecol. 1-11. https://doi.org/10.1007/s00248-018-1288-8

7. Costa S, Lopes I, Proença DN, et al (2016) Diversity of cutaneous microbiome of Pelophylax perezi populations inhabiting different environments. Sci. Total Environ. 572:995-1004. https://doi.org/10.1016/j.scitotenv.2016.07.230

8. Varga JFA, Bui-Marinos MP, Katzenback BA (2019) Frog skin innate immune defences: Sensing and surviving pathogens. Front. Immunol. 10:3128. https://doi.org/10.3389/fimmu.2018.03128

9. Woodhams DC, Vredenburg VT, Simon MA, et al (2007) Symbiotic bacteria contribute to innate immune defenses of the threatened mountain yellow-legged frog, Rana muscosa. Biol. Conserv. 138:390-398. https://doi.org/10.1016/j.biocon.2007.05.004

10. Harris RN, Brucker RM, Walke JB, et al (2009) Skin microbes on frogs prevent morbidity and mortality caused by a lethal skin fungus. ISME J 3:818-824. https://doi.org/10.1038/ismej.2009.27 
635 11. Brucker RM, Baylor CM, Walters RL, et al (2008) The identification of 2,4diacetylphloroglucinol as an antifungal metabolite produced by cutaneous bacteria of the salamander Plethodon cinereus. J Chem. Ecol. 34:39-43. https://doi.org/10.1007/s10886007-9352-8

12. Lauer A, Simon MA, Banning JL, et al (2007) Common Cutaneous Bacteria from the Eastern Red-Backed Salamander Can Inhibit Pathogenic Fungi. Copeia 2007:630-640. https://doi.org/10.1643/0045-8511(2007)2007[630:CCBFTE]2.0.CO;2

643

13. Lauer A, Simon MA, Banning JL, et al (2008) Diversity of cutaneous bacteria with antifungal activity isolated from female four-toed salamanders. ISME J 2:145-157. https://doi.org/10.1038/ismej.2007.110

14. Daszak P, Cunningham AA, Hyatt AD (2003) Infectious disease and amphibian population declines. Divers. Distrib. 9:141-150.

https://doi.org/10.1046/j.1472-4642.2003.00016.x

15. Kilpatrick AM, Briggs CJ, Daszak P (2010) The ecology and impact of chytridiomycosis: an emerging disease of amphibians. Trends Ecol. Evol. 25:109-118. https://doi.org/10.1016/j.tree.2009.07.011

16. Harris RN, Lauer A, Simon MA, et al (2009) Addition of antifungal skin bacteria to salamanders ameliorates the effects of chytridiomycosis. Dis. Aquat. Organ. 83:11-16. https://doi.org/10.3354/dao02004

17. Kueneman JG, Woodhams DC, Harris R, et al (2016) Probiotic treatment restores protection against lethal fungal infection lost during amphibian captivity. Proc. R. Soc. B. Biol. Sci. 283:20161553. https://doi.org/10.1098/rspb.2016.1553

18. Muletz-Wolz CR, Almario JG, Barnett SE, et al (2017) Inhibition of fungal pathogens across genotypes and temperatures by amphibian skin bacteria. Front. Microbiol. 8:1551. https://doi.org/10.3389/fmicb.2017.01551

19. Ellison S, Knapp RA, Sparagon W, et al (2019) Reduced skin bacterial diversity correlates with increased pathogen infection intensity in an endangered amphibian host. Mol. Ecol. 28:127-140. https://doi.org/10.1111/mec.14964

20. Walke JB, Becker MH, Loftus SC, et al (2015) Community structure and function of amphibian skin microbes: An experiment with bullfrogs exposed to a chytrid fungus. PLoS One 10:e0139848. https://doi.org/10.1371/journal.pone.0139848

21. Campbell LJ, Garner TWJ, Hopkins K, et al (2019) Outbreaks of an Emerging Viral Disease Covary With Differences in the Composition of the Skin Microbiome of a Wild United Kingdom Amphibian. Front. Microbiol. 10:1245. https://doi.org/10.3389/fmicb.2019.01245

22. Berven KA, Gill DE (1983) Interpreting geographic variation in life-history traits. Integr. Comp. Biol. 23:85-97. https://doi.org/10.1093/icb/23.1.85

23. Berven KA (1990) Factors affecting population fluctuations in larval and adult stages of the wood frog (Rana sylvatica). Ecology 71:1599-1608. https://doi.org/10.2307/1938295

24. Matutte B, Storey KB, Knoop FC, Conlon JM (2000) Induction of synthesis of an antimicrobial peptide in the skin of the freeze-tolerant frog, Rana sylvatica, in response to environmental stimuli. FEBS Lett. 483:135-138. https://doi.org/10.1016/S00145793(00)02102-5

679

25. Storey KB, Storey JSKM (1992) Natural Freeze Tolerance In Ectothermic Vertebrates. Annu. Rev. Physiol. 54:619-637. https://doi.org/10.1146/annurev.physiol.54.1.619 
680 26. Gahl MK, Longcore JE, Houlahan JE (2012) Varying Responses of Northeastern North American Amphibians to the Chytrid Pathogen Batrachochytrium dendrobatidis. Conserv. Biol. 26:135-141. https://doi.org/10.1111/j.1523-1739.2011.01801.x

684

685

27. Forzán MJ, Jones KM, Ariel E, et al (2017) Pathogenesis of Frog Virus 3 (Ranavirus, Iridoviridae) Infection in Wood Frogs (Rana sylvatica). Vet. Pathol. 54:531-548. https://doi.org/10.1177/0300985816684929

28. Becker MH, Walke JB, Cikanek S, et al (2015) Composition of symbiotic bacteria predicts survival in Panamanian golden frogs infected with a lethal fungus. Proc. R. Soc. B. Biol. Sci. 282:20142881-20142881. https://doi.org/10.1098/rspb.2014.2881

29. Rebollar EA, Gutiérrez-Preciado A, Noecker C, et al (2018) The skin microbiome of the neotropical frog Craugastor fitzingeri: Inferring potential bacterial-host-pathogen interactions from metagenomic data. Front. Microbiol. 9:466. https://doi.org/10.3389/fmicb.2018.00466

30. Walters W, Hyde ER, Berg-Lyons D, et al (2016) Improved Bacterial 16S rRNA Gene (V4 and V4-5) and Fungal Internal Transcribed Spacer Marker Gene Primers for Microbial Community Surveys. mSystems 1:e00009-15. https://doi.org/10.1128/mSystems.00009-15

31. Apprill A, Mcnally S, Parsons R, Weber L (2015) Minor revision to V4 region SSU rRNA 806R gene primer greatly increases detection of SAR11 bacterioplankton. Aquat. Microb. Ecol. 75:129-137. https://doi.org/10.3354/ame01753

32. Parada AE, Needham DM, Fuhrman JA (2016) Every base matters: Assessing small subunit rRNA primers for marine microbiomes with mock communities, time series and global field samples. Environ. Microbiol. 18:1403-1414. https://doi.org/10.1111/14622920.13023

33. Bolyen E, Rideout JR, Dillon MR, et al (2019) Reproducible, interactive, scalable and extensible microbiome data science using QIIME 2. Nat. Biotechnol. 37:852-857. https://doi.org/10.1038/s41587-019-0209-9

34. Callahan BJ, McMurdie PJ, Rosen MJ, et al (2016) DADA2: High-resolution sample inference from Illumina amplicon data. Nat. Methods. 13:581-583. https://doi.org/10.1038/nmeth.3869

35. Yilmaz P, Parfrey LW, Yarza P, et al (2014) The SILVA and "all-species Living Tree Project (LTP)" taxonomic frameworks. Nucleic Acids Res. 42:D643-D648. https://doi.org/10.1093/nar/gkt1209

36. Katoh, K., Misawa, K., Kuma, K., Miyata T (2002) MAFFT: a novel method for rapid multiple sequence alignment based on fast Fourier transform. Nucleic Acids Res. 30:3059-3066. https://doi.org/10.1093/nar/gkf436

37. Stamatakis A (2014) RAxML version 8: A tool for phylogenetic analysis and postanalysis of large phylogenies. Bioinformatics 30:1312-1313. https://doi.org/10.1093/bioinformatics/btu033

38. Medina D, Hughey MC, Becker MH, et al (2017) Variation in Metabolite Profiles of Amphibian Skin Bacterial Communities Across Elevations in the Neotropics. Microb. Ecol. 74:227-238. https://doi.org/10.1007/s00248-017-0933-y

39. Shade A, Handelsman J (2012) Beyond the Venn diagram: The hunt for a core microbiome. Environ. Microbiol. 14:4-12. https://doi.org/10.1111/j.14622920.2011.02585.x 
40. Woodhams DC, Alford RA, Antwis RE, et al (2015) Antifungal isolates database of amphibian skin-associated bacteria and function against emerging fungal pathogens. Ecology 96:595-595. https://doi.org/10.1890/14-1837.1

41. Walke JB, Becker MH, Loftus SC, et al (2014) Amphibian skin may select for rare environmental microbes. ISME J 8:2207-2217. https://doi.org/10.1038/ismej.2014.77

42. Salter SJ, Cox MJ, Turek EM, et al (2014) Reagent and laboratory contamination can critically impact sequence-based microbiome analyses. BMC Biol. 12:87. https://doi.org/10.1186/s12915-014-0087-z

43. Berven KA, Grudzien TA (1990) Dispersal in the Wood Frog (Rana sylvatica): Implications for Genetic Population Structure. Evolution (N Y) 44:2047-2056. https://doi.org/10.2307/2409614

44. Prado-Irwin SR, Bird AK, Zink AG, Vredenburg VT (2017) Intraspecific Variation in the Skin-Associated Microbiome of a Terrestrial Salamander. Microb. Ecol. 74:745-756. https://doi.org/10.1007/s00248-017-0986-y

45. Muletz Wolz CR, Yarwood SA, Campbell Grant EH, et al (2018) Effects of host species and environment on the skin microbiome of Plethodontid salamanders. J. Anim. Ecol. 87:341-353. https://doi.org/10.1111/1365-2656.12726

46. Bletz MC, Archer H, Harris RN, et al (2017) Host ecology rather than host phylogeny drives amphibian skin microbial community structure in the biodiversity hotspot of Madagascar. Front. Microbiol. 8:1530. https://doi.org/10.3389/fmicb.2017.01530

47. Albecker MA, Belden LK, McCoy MW (2018) Comparative Analysis of Anuran Amphibian Skin Microbiomes Across Inland and Coastal Wetlands. Microb. Ecol. 78:348. https://doi.org/10.1007/s00248-018-1295-9

48. Fierer N, Hamady M, Lauber CL, Knight R (2008) The influence of sex, handedness, and washing on the diversity of hand surface bacteria. Proc. Natl. Acad. Sci. 105:1799417999. https://doi.org/10.1073/pnas.0807920105

49. Saag P, Tilgar V, Mänd R, et al (2011) Plumage Bacterial Assemblages in a Breeding Wild Passerine: Relationships with Ecological Factors and Body Condition. Microb. Ecol. 61:740-749. https://doi.org/10.1007/s00248-010-9789-0

50. Kielak AM, Barreto CC, Kowalchuk GA, et al (2016) The ecology of Acidobacteria: Moving beyond genes and genomes. Front. Microbiol. 7:744 https://doi.org/10.3389/fmicb.2016.00744

51. Grönroos M, Parajuli A, Laitinen OH, et al (2019) Short-term direct contact with soil and plant materials leads to an immediate increase in diversity of skin microbiota. MicrobiologyOpen 8:e00645. https://doi.org/10.1002/mbo3.645

52. Zaheer R, Noyes N, Ortega Polo R, et al (2018) Impact of sequencing depth on the characterization of the microbiome and resistome. Sci. Rep. 8:5890. https://doi.org/10.1038/s41598-018-24280-8

53. McMurdie PJ, Holmes S (2014) Waste Not, Want Not: Why Rarefying Microbiome Data Is Inadmissible. PLoS Comput. Biol. 10:e1003531. https://doi.org/10.1371/journal.pcbi.1003531 


\section{Figure Legends}

Fig. 1 Comparison of frog swab and pond water $\alpha$-diversity metrics. Sample $\alpha$-diversity was

770 calculated using a sampling depth of 10,000. Mean seasonal value and standard deviation of each

771 group is shown . Results are given for (a) ASV Richness, (b) Shannon's Diversity Index and (c)

772 Faith's Phylogenetic Diversity

773

774 Fig. 2 Relative frequency of microbial phyla ASVs present in individual R. sylvatica skin swab

775 samples and controls. Phyla are listed from top to bottom in order of decreasing summed total

776 ASV frequency. Bars represent relative frequency within a sample and are given in

777 corresponding order

778

779 Fig. 3 Principal coordinate analysis of $\beta$-diversity of spring frog skin and pond water 780 microbiome samples. Principal coordinate analysis plots were created using Emperor from 781 distance matrices calculated using a sampling depth of 10,000. Plots were limited to representing

782 the two dimensions with the highest percent variation explained and were calculated for (a)

783 Unweighted UniFrac distances, (b) Weighted UniFrac distances, and (c) Bray-Curtis distances

785 Fig. 4 Comparison of seasonal wood frog skin microbiota $\alpha$-diversity metrics. Sample $\alpha$ 786 diversity was calculated using a sampling depth of 10,000. Mean seasonal value and standard

787 deviation of each group is shown. Results are given for (a) ASV Richness, (b) Shannon's 788 Diversity Index and (c) Faith's Phylogenetic Diversity 
790 Fig. 5 Principal coordinate analysis of $\beta$-diversity of frog skin microbiome samples. Principal

791 coordinate analysis plots were created using Emperor from distance matrices calculated using a

792 sampling depth of 10,000 . Plots were limited to representing the two dimensions with the highest

793 percent variation explained and were calculated for (a) Unweighted UniFrac distances, (b)

794 Weighted UniFrac distances, and (c) Bray-Curtis distances

795

796 Fig. 6 Relative abundance of core microbial families in wood frog skin microbiota. Families

797 included were present in $\geq 90 \%$ of all frog skin samples and had a mean relative abundance $\geq 1 \%$.

798 Abundance of each family was compared between seasonal groups using pair-wise ANOVA,

799 letters are used to indicate significant inter-seasonal variation for a given family. Seasons marked

800 with the same letter do not significantly differ.

801

802 Fig. 7 Overlap of core microbial families present on wood frog skin across seasons. Core taxa

803 were defined as those found in $90 \%$ or more samples from a given season. Taxa were combined

804 at the level of (a) genus and (b) family, omitting entries with ambiguous taxonomy 


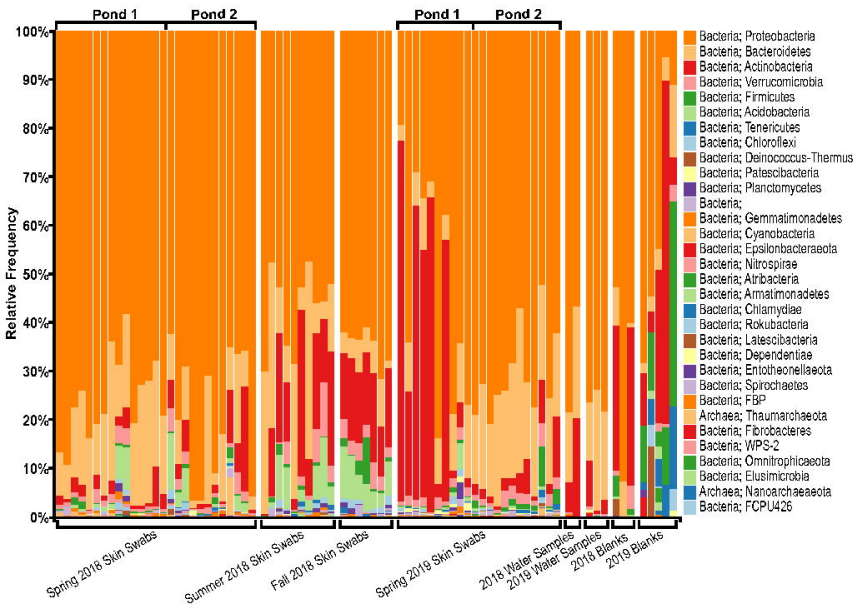




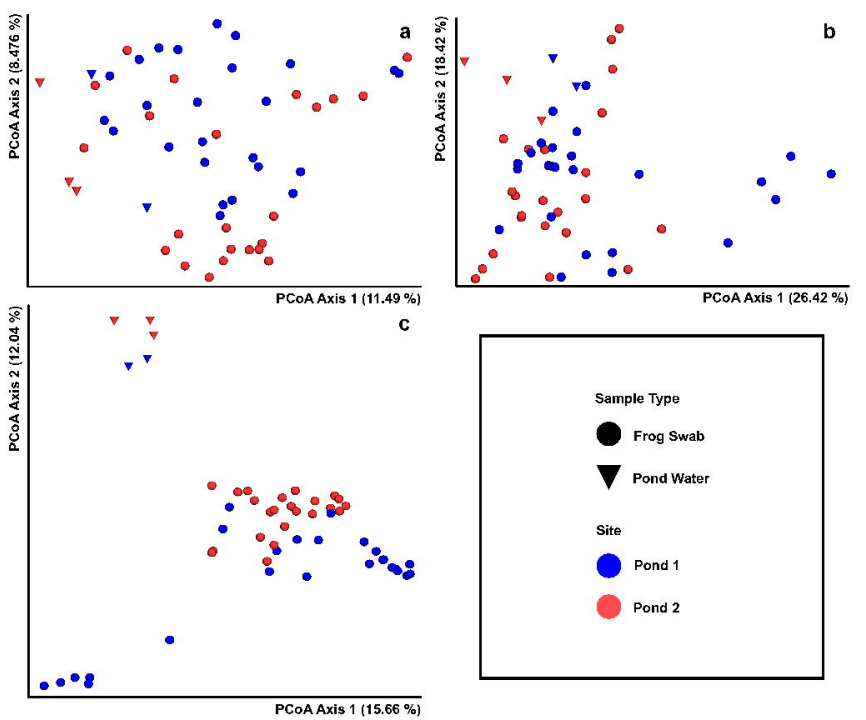




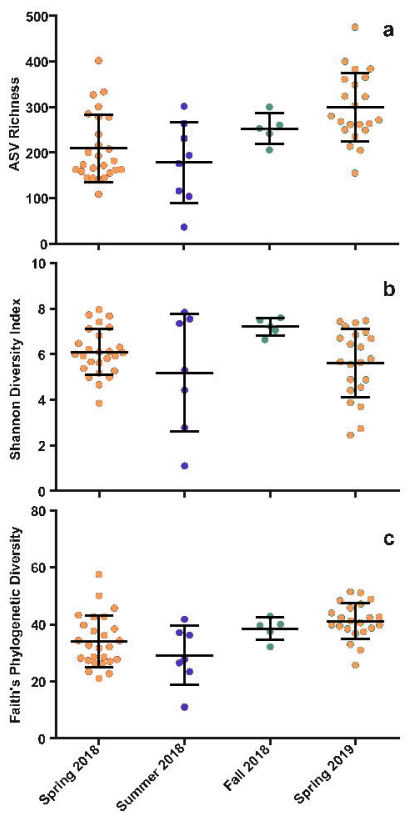




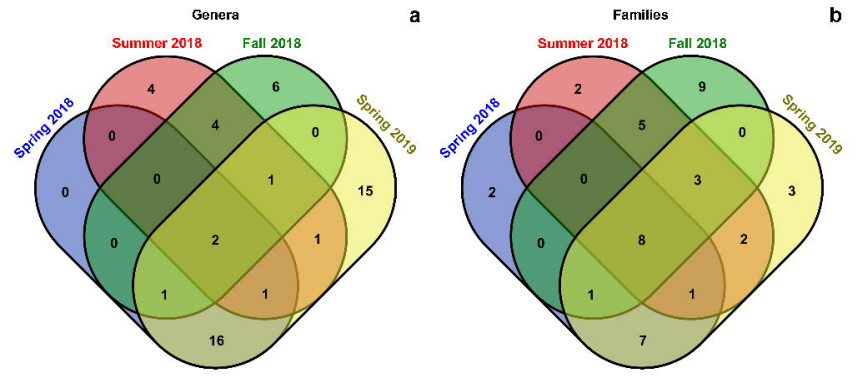


Table 1 Summary of frog skin swab samples. Season, site and sex of frog are given for each skin swab sample. Male frogs are denoted with an "M", while females are denoted with an "F"

\begin{tabular}{|cccc|}
\hline Season & Pond 1 & $\begin{array}{c}\text { Capture Site } \\
\text { Pond 2 }\end{array}$ & Forest Floor \\
\hline Spring 2018 & $13 \mathrm{M} / 2 \mathrm{~F}$ & $8 \mathrm{M} / 4 \mathrm{~F}$ & \\
Summer 2018 & & & $11 \mathrm{M} / \mathrm{O} \mathrm{F}$ \\
Fall 2018 & & & $5 \mathrm{M} / 2 \mathrm{~F}$ \\
Spring 2019 & $10 \mathrm{M} / 0 \mathrm{~F}$ & $12 \mathrm{M} / 0 \mathrm{~F}$ & \\
\hline
\end{tabular}


Table 2 Summary of adonis (PERMANOVA) models of $\beta$-diversity for microbial communities on frog skin during spring months and in pond water samples. Effects on variation due to sample type (frog skin, water), site (Pond 1, Pond 2) and year (2018, 2019) are considered. Significant results are marked with an asterisk

\begin{tabular}{|c|c|c|c|c|c|c|}
\hline Variables & $\begin{array}{l}\text { Degrees of } \\
\text { Freedom }\end{array}$ & $\begin{array}{l}\text { Sums of } \\
\text { Squares }\end{array}$ & $\begin{array}{c}\text { Mean } \\
\text { Squares }\end{array}$ & F.Model & $\mathbf{R}^{2}$ & $\mathbf{p}$ \\
\hline \multicolumn{7}{|c|}{ a) Unweighted UniFrac Distance } \\
\hline Sample Type & 1 & 0.69 & 0.69 & 4.08 & 0.07 & $0.001^{*}$ \\
\hline Site & 1 & 0.48 & 0.48 & 2.85 & 0.05 & $0.001^{*}$ \\
\hline Year & 1 & 0.70 & 0.70 & 4.20 & 0.07 & $0.001^{*}$ \\
\hline Residuals & & 8.23 & 0.17 & & 0.81 & \\
\hline Total & & 10.10 & & & 1.00 & \\
\hline \multicolumn{7}{|c|}{ b) Weighted UniFrac Distance } \\
\hline Sample Type & 1 & 0.66 & 0.66 & 6.86 & 0.11 & $0.001^{*}$ \\
\hline Site & 1 & 0.30 & 0.30 & 3.13 & 0.05 & $0.005^{\star}$ \\
\hline Year & 1 & 0.44 & 0.44 & 4.54 & 0.07 & $0.001^{*}$ \\
\hline Residuals & & 4.70 & 0.10 & & 0.77 & \\
\hline Total & & 6.10 & & & 1.00 & \\
\hline \multicolumn{7}{|c|}{ c) Bray-Curtis Dissimilarity } \\
\hline Sample Type & 1 & 1.83 & 1.83 & 7.71 & 0.12 & $0.001^{*}$ \\
\hline Site & 1 & 1.12 & 1.12 & 4.70 & 0.07 & $0.001^{*}$ \\
\hline Year & 1 & 1.28 & 1.28 & 5.39 & 0.08 & $0.001^{*}$ \\
\hline Residuals & & 11.64 & 0.24 & & 0.73 & \\
\hline Total & & 15.87 & & & 1.00 & \\
\hline
\end{tabular}


Table 3 Summary of adonis (PERMANOVA) models of $\beta$-diversity for microbial communities on frog skin swab samples. Effects on variation due to season (spring, summer, fall) are considered. Significant results are marked with an asterisk

\begin{tabular}{|c|c|c|c|c|c|c|}
\hline Variables & $\begin{array}{c}\text { Degrees of } \\
\text { Freedom }\end{array}$ & $\begin{array}{l}\text { Sums of } \\
\text { Squares }\end{array}$ & $\begin{array}{c}\text { Mean } \\
\text { Squares }\end{array}$ & F.Model & $\mathbf{R}^{2}$ & $\mathbf{p}$ \\
\hline \multicolumn{7}{|c|}{ a) Unweighted UniFrac Distance } \\
\hline Season & 2 & 1.61 & 0.80 & 4.36 & 0.13 & $0.001^{*}$ \\
\hline Residuals & & 10.51 & 0.18 & & 0.87 & \\
\hline Total & & 12.12 & & & 1.00 & \\
\hline \multicolumn{7}{|c|}{ b) Weighted UniFrac Distance } \\
\hline Season & 2 & 1.73 & 0.86 & 7.04 & 0.20 & $0.001^{*}$ \\
\hline Residuals & & 6.99 & 0.12 & & 0.80 & \\
\hline Total & & 8.72 & & & 1.00 & \\
\hline \multicolumn{7}{|c|}{ c) Bray-Curtis Dissimilarity } \\
\hline Season & 2 & 3.59 & 1.79 & 6.17 & 0.18 & $0.001^{*}$ \\
\hline Residuals & & 16.57 & 0.29 & & 0.82 & \\
\hline Total & & 20.15 & & & 1.00 & \\
\hline
\end{tabular}


Table 4 Core microbiome genera and their mean relative abundance. Listed genera are present in $\geq 90 \%$ of all frog skin swab samples. If a microbial genus was not core to every seasonal group ("All”), seasonal groups for which the microbial genera was present in $\geq 90 \%$ of individual frog skin swab samples are listed. Wood frog skin swabs collected during different seasons are denoted in abbreviated form [spring (Sp), summer $(\mathrm{Su})$ and fall (Fa) and corresponding year ((20)18 or 19)]

\begin{tabular}{|cccc|}
\hline Genera & Phylum & $\begin{array}{c}\text { Mean Relative } \\
\text { Abundance }\end{array}$ & Seasonally Core \\
\hline Ferruginobacter & Bacteroidetes & 0.009 & Sp18, Sp19 \\
Uncultured & Bacteroidetes & 0.007 & Sp18, Su18, Sp19 \\
Chitinophagaceae & Proteobacteria & 0.006 & Su18, Fa18, Sp19 \\
Methylobacterium & All \\
Allo/Neo/Para/Rhizobium & Proteobacteria & 0.008 & All \\
Sphingomonas & Proteobacteria & 0.038 & Sp18, Sp19 \\
Massilia & Proteobacteria & 0.019 & Su18, Sp19 \\
Pseudomonas & Proteobacteria & 0.026 & \\
\hline
\end{tabular}


Table 5 Core microbiome families and their mean relative abundance. Listed families are present in $\geq 90 \%$ of all frog skin swab samples. If a microbial family was not core to every seasonal group (“All”), seasonal groups for which the microbial family was present in $\geq 90 \%$ of individual frog skin swab samples are listed. Wood frog skin swabs collected during different seasons are denoted in abbreviated form [spring (Sp), summer ( $\mathrm{Su})$ and fall $(\mathrm{Fa})$ and corresponding year ((20)18 or 19)]

\begin{tabular}{|clcc|}
\hline Family & Phylum & $\begin{array}{c}\text { Mean Relative } \\
\text { Abundance }\end{array}$ & Seasonally Core \\
\hline Chitinophagaceae & Bacteroidetes & 0.019 & All \\
Spirosomaceae & Bacteroidetes & 0.037 & Sp18, Sp19 \\
Sphingobacteriaceae & Bacteroidetes & 0.039 & Sp18, Su18, Sp19 \\
Acetobacteraceae & Proteobacteria & 0.009 & Su18, Fa18, Sp19 \\
Caulobacteraceae & Proteobacteria & 0.014 & All \\
Beijerinckiaceae & Proteobacteria & 0.014 & All \\
Rhizobiaceae & Proteobacteria & 0.013 & All \\
Xanthobacteraceae & Proteobacteria & 0.021 & All \\
Sphingomonadaceae & Proteobacteria & 0.062 & All \\
Burkholderiaceae & Proteobacteria & 0.329 & All \\
Xanthomonadaceae & Proteobacteria & 0.00401 & All \\
\hline
\end{tabular}


Table 6 Putatively antifungal genera and their seasonal relative abundances. Genera which are found on $R$. sylvatica skin and have antifungal isolates listed in the Antifungal Isolates Database (Woodhams et al., 2015) are listed. Darker shading indicates greater relative abundance

\begin{tabular}{|c|c|c|c|c|}
\hline \multirow[b]{2}{*}{ Genus } & \multicolumn{4}{|c|}{ Mean Relative Abundance } \\
\hline & $\begin{array}{l}\text { Spring } \\
2018\end{array}$ & $\begin{array}{l}\text { Summer } \\
2018\end{array}$ & $\begin{array}{l}\text { Fall } \\
2018\end{array}$ & $\begin{array}{c}\text { Spring } \\
2019\end{array}$ \\
\hline Acinetobacter & 0.008 & 0.004 & 0.000 & 0.000 \\
\hline Aeromonas & 0.003 & 0.001 & 0.001 & 0.000 \\
\hline $\begin{array}{l}\text { Allorhizobium-Neorhizobium- } \\
\text { Pararhizobium-Rhizobium }\end{array}$ & 0.008 & 0.006 & 0.014 & 0.007 \\
\hline Bacillus & 0.000 & 0.001 & 0.002 & 0.000 \\
\hline Brevundimonas & 0.010 & 0.001 & 0.001 & 0.006 \\
\hline $\begin{array}{l}\text { Burkholderia-Caballeronia- } \\
\text { Paraburkholderia }\end{array}$ & 0.000 & 0.006 & 0.051 & 0.001 \\
\hline Chryseobacterium & 0.015 & 0.001 & 0.000 & 0.016 \\
\hline Comamonas & 0.000 & 0.001 & 0.001 & 0.000 \\
\hline Curtobacterium & 0.004 & 0.013 & 0.003 & 0.162 \\
\hline Duganella & 0.011 & 0.001 & 0.002 & 0.006 \\
\hline Dyella & 0.000 & 0.001 & 0.010 & 0.000 \\
\hline Flavobacterium & 0.015 & 0.000 & 0.008 & 0.029 \\
\hline Janthinobacterium & 0.003 & 0.000 & 0.000 & 0.003 \\
\hline Lactococcus & 0.000 & 0.000 & 0.000 & 0.000 \\
\hline Lysobacter & 0.000 & 0.001 & 0.001 & 0.000 \\
\hline Massilia & 0.030 & 0.002 & 0.004 & 0.018 \\
\hline Micrococcus & 0.000 & 0.002 & 0.000 & 0.000 \\
\hline Novosphingobium & 0.006 & 0.002 & 0.003 & 0.009 \\
\hline Paenibacillus & 0.005 & 0.006 & 0.007 & 0.005 \\
\hline Pantoea & 0.000 & 0.001 & 0.000 & 0.000 \\
\hline Pedobacter & 0.007 & 0.003 & 0.003 & 0.019 \\
\hline Polaromonas & 0.003 & 0.000 & 0.003 & 0.009 \\
\hline Pseudomonas & 0.012 & 0.009 & 0.037 & 0.037 \\
\hline Pseudoxanthomonas & 0.000 & 0.000 & 0.000 & 0.000 \\
\hline Serratia & 0.000 & 0.000 & 0.000 & 0.000 \\
\hline Silvimonas & 0.000 & 0.000 & 0.003 & 0.000 \\
\hline Sphingobacterium & 0.000 & 0.001 & 0.000 & 0.000 \\
\hline Staphylococcus & 0.001 & 0.004 & 0.017 & 0.000 \\
\hline Stenotrophomonas & 0.000 & 0.001 & 0.006 & 0.001 \\
\hline Streptomyces & 0.001 & 0.001 & 0.005 & 0.000 \\
\hline Undibacterium & 0.007 & 0.000 & 0.000 & 0.009 \\
\hline Variovorax & 0.005 & 0.006 & 0.007 & 0.005 \\
\hline
\end{tabular}

